\title{
Magnetic Bead-Based Immunoassay Allows Rapid, Inexpensive, and Quantitative Detection of Human SARS-CoV-2 Antibodies
}

\author{
Luciano F. Huergo,* Khaled A. Selim, Marcelo S. Conzentino, Edileusa C. M. Gerhardt, \\ Adrian R. S. Santos, Berenike Wagner, Janette T. Alford, Nelli Deobald, Fabio O. Pedrosa, \\ Emanuel M. de Souza, Meri B. Nogueira, Sônia M. Raboni, Dênio Souto, Fabiane G. M. Rego, \\ Dalila L. Zanette, Mateus N. Aoki, Jeanine M. Nardin, Bruna Fornazari, Hugo M. P. Morales, \\ Vânia A. Borges, Annika Nelde, Juliane S. Walz, Matthias Becker, Nicole Schneiderhan-Marra, \\ Ulrich Rothbauer, Rodrigo A. Reis, and Karl Forchhammer
}

Cite This: https://dx.doi.org/10.1021/acssensors.0c02544

Read Online

ABSTRACT: Immunological methods to detect SARS-CoV-2 seroconversion in humans are important to track COVID-19 cases and the humoral response to SARS-CoV-2 infections and immunization to future vaccines. The aim of this work was to develop a simple chromogenic magnetic bead-based immunoassay which allows rapid, inexpensive, and quantitative detection of human antibodies against SARS-CoV-2 in serum, plasma, or blood. Recombinant 6xHis-tagged SARS-CoV-2 Nucleocapsid protein was mobilized on the surface of $\mathrm{Ni}^{2+}$ magnetic beads and challenged with serum or blood samples obtained from controls or COVID-19 cases. The beads were washed, incubated with anti-human IgG-HPR conjugate, and immersed into a solution containing a chromogenic

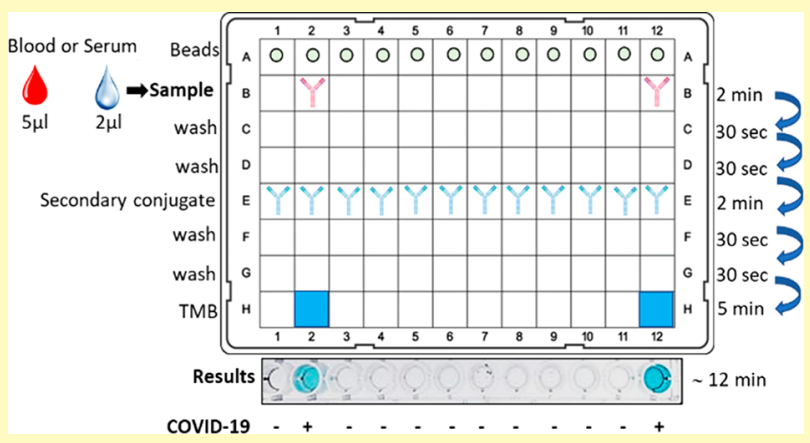
HPR substrate. Bead transfer and homogenization between solutions was aided by a simple low-cost device. The method was validated by two independent laboratories, and the performance to detect SARS-CoV-2 seroconversion in humans was in the same range as obtained using the gold standard immunoassays ELISA and Luminex, though requiring only a fraction of consumables, instrumentation, time to deliver results, and volume of sample. Furthermore, the results obtained with the method described can be visually interpreted without compromising accuracy as demonstrated by validation at a point-of-care unit. The magnetic bead immunoassay throughput can be customized on demand and is readily adapted to be used with any other 6xHis tagged protein or peptide as antigen to track other diseases.

KEYWORDS: COVID-19, magnetic ELISA, SARS-CoV-2, immunological method, magnetic beads

7 he COVID-19 immunological tests detect antibodies
reacting against SARS-COV-2 antigens. ${ }^{1}$ One of the main advantages of immunological tests is that they can be used to screen COVID-19 cases after the acute phase of the disease given that antibodies remain detectable for months after onset of symptoms or viral detection by RT-PCR., ${ }^{2,3}$ Hence, COVID19 immunological tests are important tools for epidemiological surveillance, analyzing the efficiency of future COVID-19 vaccines and humoral response kinetics to SARS-CoV-2 infections and/or immunization. ${ }^{4}$ Furthermore, COVID-19 immunological tests may act as an additional toolkit to identify COVID-19 in the acute phase of the disease given that some patients produce detectable antibodies as early as 2 days after symptom onset. ${ }^{2,3}$

The ELISA assay (enzyme-linked immunosorbent assay), described by Engvall and Perlmann in the early seventies, ${ }^{5}$ is still considered the gold standard immunological method, as it can provide a low-cost, easy to implement, and highthroughput method to quantitatively detect antibodies. ${ }^{6}$ Indeed, ELISA assays have been used to successfully detect SARS-CoV-2 seroconversion in humans. ${ }^{7,8}$ The main shortcoming of ELISA is that it is not suited to point-of-care analysis. On the other hand, lateral flow immunochromatography, which has been widely used to track COVID-19 cases, is relatively inexpensive and can be applied for point-of-care, providing the results in 15-20 min. The shortcomings of immunochromatography are that it only provides qualitative

Received: December 4, 2020

Accepted: January 20, 2021 
SARS-CoV-2 $\mathrm{N}$ protein

coated magnetic beads

Added to all wells lane A
Human IgG reacting with $N$ protein

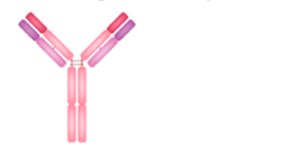

Present in COVID-19 serum converted patients
Anti human IgG-HPR

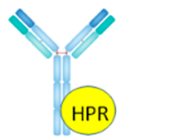

Added to all wells lane $E$

b
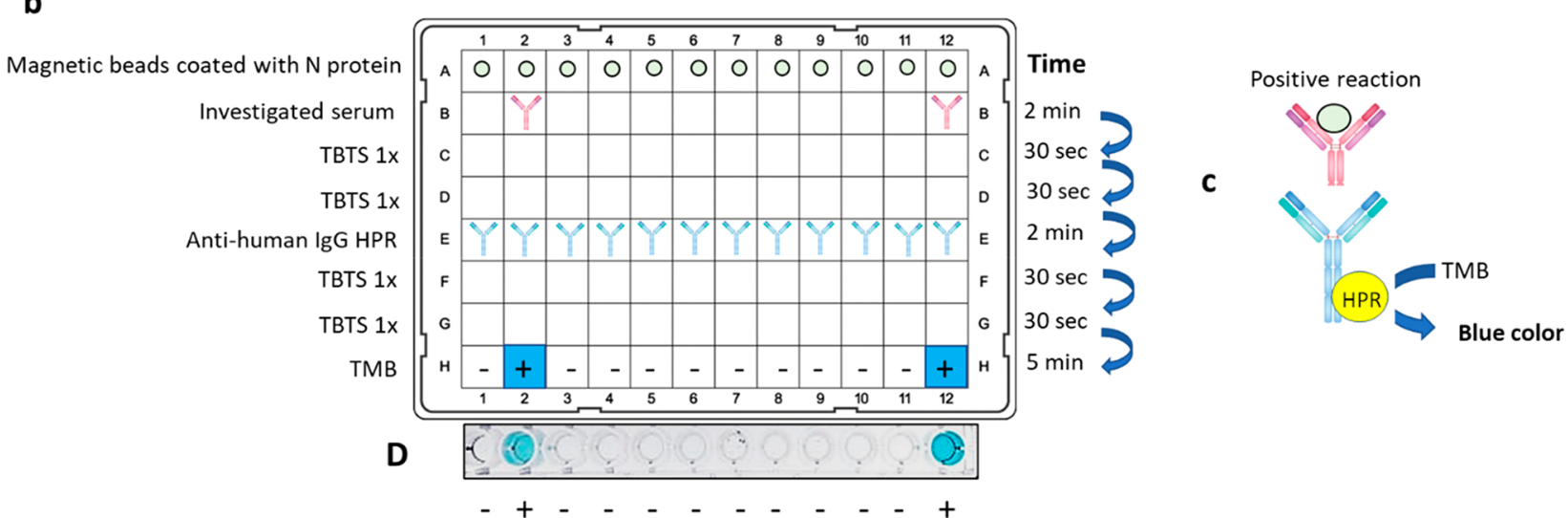

Figure 1. Magnetic bead-based chromogenic ELISA. (a) Reaction components. (b) Diagram of the reaction and distribution of the components on a 96 well plate. (b) Positive samples will develop a blue color in line $\mathrm{H}$ due to the formation of a ternary complex between antigen and primary and HPR secondary antibody. (d) Photography register of a typical assay. H2 and H12, serum from two PCR-confirmed patients (mild and severe, respectively). Other lanes serum from negative controls.

data and is not adaptable to high-throughput analysis, and the accuracy of some COVID-19 tests based on this method available on the market are questionable. ${ }^{10}$

\section{EXPERIMENTAL SECTION}

Antigen Purification. ELISA assays and human samples: Details of antigen production and purification, ELISA assays, and human samples are provided in Supporting Information.

Magnetic Bead-Based Immunoassay. The magnetic beadbased immunoassay was developed using MagneHis $\mathrm{Ni}^{2+}$ magnetic beads (Promega cat number V8550) as described previously. ${ }^{6} 1 \mathrm{~mL}$ of the beads was washed $2 \times$ with $1 \mathrm{~mL}$ of TBST and resuspended in 50 $\mathrm{mL}$ of TBST containing $0.75 \mathrm{mg}$ of purified His-tagged N-protein. The beads and antigen were incubated for $10 \mathrm{~min}$ at room temperature with gentle mixing. The beads were washed with 20 $\mathrm{mL}$ of TBST and resuspended in $5 \mathrm{~mL}$ of TBST. The antigen coated beads where separated in $0.1 \mathrm{~mL}$ aliquots, which were kept in the fridge up to 3 months. For use, $0.1 \mathrm{~mL}$ aliquots were resuspended in $1.2 \mathrm{~mL}$ of TBST containing $1 \%(\mathrm{w} / \mathrm{v})$ skimmed milk, and $0.1 \mathrm{~mL}$ aliquots were distributed into each well of a 96 well plate (Figure 1). 2 $\mu \mathrm{L}$ of human serum (or $5 \mu \mathrm{L}$ of blood) were diluted in $0.2 \mathrm{~mL}$ of TBST $1 \times$ skimmed milk $1 \%(\mathrm{w} / \mathrm{v})$ directly in the wells of the 96 well plate. The magnetic beads coated with $\mathrm{N}$ protein were mixed with the diluted serum for $2 \mathrm{~min}$. The beads were captured using a simple homemade magnetic extractor device (Figure S2 and SI video) and loaded into sequential $2 \times$ wash steps for $30 \mathrm{~s}$ in $1 \times$ TBST. The beads were incubated for $2 \mathrm{~min}$ with $0.15 \mathrm{~mL}$ goat anti-human IgG-HPR (Thermo Scientific) diluted 1:3000 in $1 \times$ TBST, following by $2 \times$ wash steps for $30 \mathrm{~s}$ TBST $1 \times$. The beads were transferred to wells with $0.15 \mathrm{~mL}$ of the HPR substrate TMB (Thermo Scientific) and 5 min. The total time of the procedure took less than $12 \mathrm{~min}$. We routinely performed the test in a 12 sample format (Figure 1 ). When the reactions were complete, the beads were removed, and the plates were put on the top of a white light transilluminator device and photographed. The optical density was measured at $650 \mathrm{~nm}$ using a TECAN M Nano plate reader (TECAN) monochromator at bandwidth $9 \mathrm{~nm}$ and 25 flashes. More details are provided in Supporting Information.
Magnetic Bead Extractor Device. A simple magnetic extractor device was prepared by fixing 12 nails $(11 \mathrm{~mm})$ on a piece of foam (Figure S2A) as described previously. ${ }^{6}$ In the head of the nails, a set of neodymium magnets were added by simple magnetic attraction. We used $2 \times(3 \times 2 \mathrm{~mm})$ and $1 \times(2 \times 2 \mathrm{~mm})$ neodymium magnets (Figure S2A). A better-quality system can be prepared using a 3D printer (Figure S2B). The extraction of the beads is performed by inserting the magnets on the PCR-strip, so the PCR-strip will hold firmly the magnetic beads during the transfer from well to well (Figure S2). Once the beads are in place in the next solution, magnets are removed, and the PCR strip is gently moved to allow proper homogenization of the magnetic beads in the solution in such way that the PCR strip acts as a mixer (SI video).

Data Analysis. The group working in Brazil used one COVID-19 positive serum as reference throughout the study. This sample showed an interassay raw OD CV\% of $8.5 \%(n=25)$ in the magnetic bead immune assay. All data were normalized as \% of the reference before applying Receiver Operating Analysis (ROC) using GraphPad Prism 7.0. For the data generated by the group working in Germany, raw OD was directly used for ROC analysis. Statistical analysis was performed using the $t$ test on GraphPad Prism 7.0.

\section{RESULTS AND DISCUSSION}

The aim of this work was to develop an inexpensive COVID19 immunological test, adaptable to both point-of-care and high-throughput diagnostic, which would provide quantitative data within minutes. The basic idea was to develop an indirect chromogenic ELISA, which functions with the antigen immobilized on the surface of magnetic beads nanoparticles (we now call magnetic bead ELISA).

As a proof of concept, we expressed and purified a Histagged version of SARS-CoV-2 Nucleocapsid $\mathrm{N}$ protein (Figure S1) and immobilized it on $\mathrm{Ni}^{2+}$ magnetic beads (antigen mobilization on beads takes about $15 \mathrm{~min}$, and precoated beads can be stored for at least 3 months in the fridge). These beads were then challenged with human serum from COVID-19 positive and control subjects for $2 \mathrm{~min}$ 

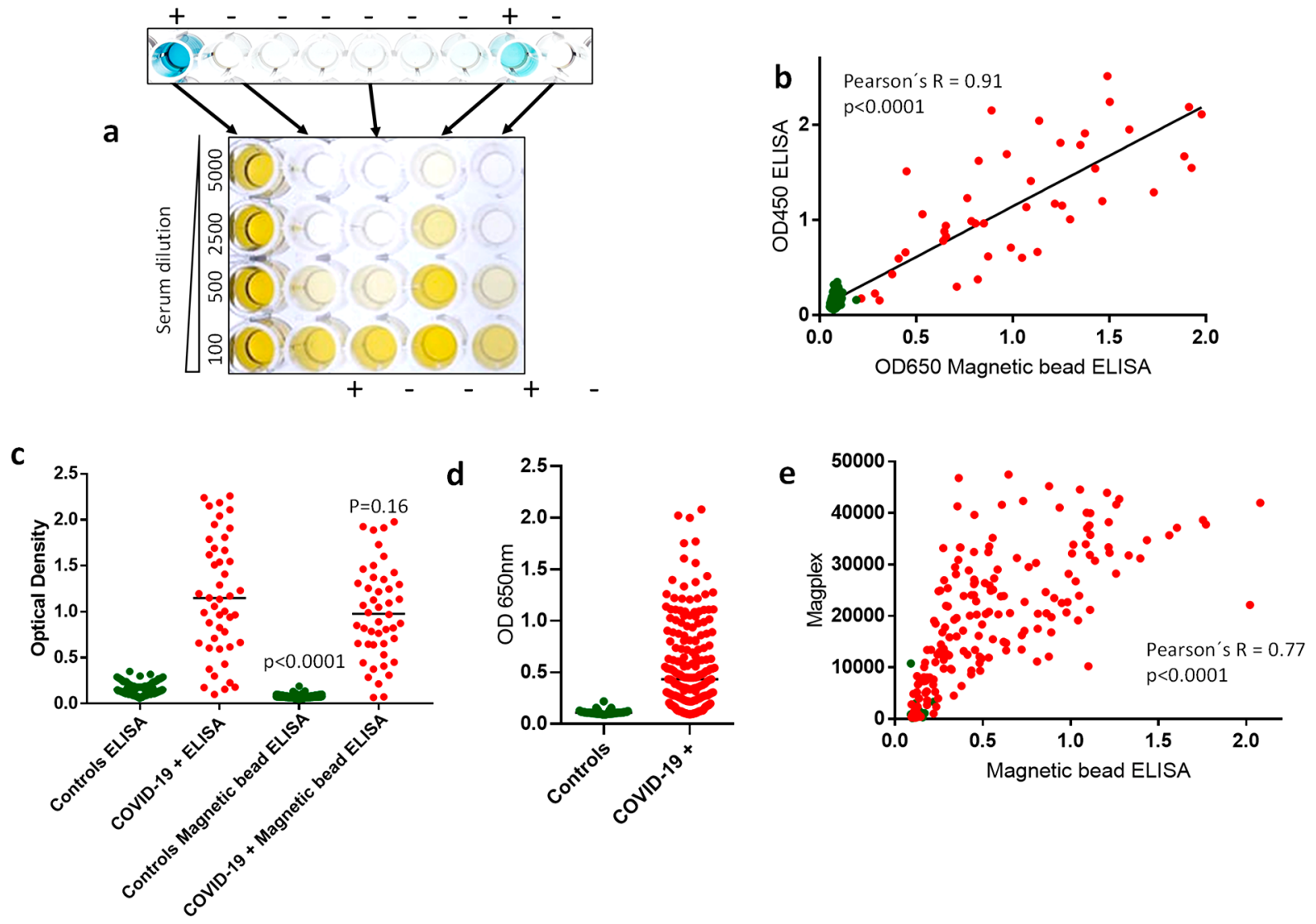

Figure 2. Comparison between classic and magnetic-bead ELISA. (a) Serum from negative controls and COVID-19 positive cases were analyzed using the magnetic bead and classic ELISA. (b) Correlation between raw OD obtained with classic vs magnetic bead ELISA. The equation for linear regression was $Y=1.06 \times X+0.08$. (c) Raw OD obtained using classic and magnetic bead ELISA. (d) Raw OD values obtained using the magnetic bead ELISA prepared by a different group of operators and different cohort of samples performed in Germany. (e) Correlation of raw fluorescence signal from Megaplex vs raw OD from magnetic bead ELISA; both systems investigated IgG against SARS-CoV-2 N protein. The equation for linear regression was $Y=25627 \times X+3236$.

following the procedure described in Figure 1 . The beads were washed two times for $30 \mathrm{~s}$, and then immersed in a solution containing anti-human IgG HPR for 2 min, followed by two additional $30 \mathrm{~s}$ washes. The beads were finally immersed in HPR chromogenic substrate and incubated for $5 \mathrm{~min}$. At the end of the assay, the beads were removed from the solution so that the results could be visually inspected. The negative controls were completely blank, whereas those of COVID-19 positive samples developed a strong blue color indicating the presence of $\operatorname{IgG}$ reacting with the SARS-CoV-2 $\mathrm{N}$ protein (Figure 1). When all reagents are in place, the procedure takes less than $12 \mathrm{~min}$ and uses only $2 \mu \mathrm{L}$ of serum at a price of consumables of less than US $\$ 1$ per sample. Effective bead transfer and homogenization between each solution was achieved using a simple in-house-built magnetic extractor/ mixer device at a cost of less than US \$2 (Figure S2 and SI video).

To confirm the efficiency of our immunological method, we compared the results obtained with those from classic ELISA, which was in house developed using polystyrene 96 well plates coated with the same SARS-CoV-2 $\mathrm{N}$ protein preparation. Serum from a COVID-19 positive case and negative control were serially diluted and allowed to react with SARS-CoV-2 N protein on both classic and magnetic bead ELISA. The COVID-19 positive serum showed strong reaction with the $\mathrm{N}$ protein, which was clearly distinguished from the negative serum (Figure S3). The raw optical density (OD) vs reciprocal dilution plot of the positive serum showed a similar profile response on both classic and magnetic bead ELISA, the dynamic ranges on both systems were equivalent with linearity observed within 6 data points of the dilution curve (Figure S3A,C). Hence, the magnetic bead ELISA can be used to provide quantitative data. The magnetic system was less sensitive than classic ELISA, 10-fold lower serum dilution was required to reach signal saturation (Figure S3A,C). However, this made the magnetic system more practical, as sera could be diluted directly on the plate of the assay bypassing the time and plastic consuming dilutions required prior classic ELISA. It worth mentioning that, whereas in classic ELISA intra-assay reproducibility was in the $8-9 \%$ range, the magnetic system showed better intraassay reproducibility (CV 2-3\%) in such way that no replicates were required, whereas they were necessary in classic ELISA (see Supporting Information for details).

The correlation of the results obtained using classic and magnetic bead ELISA was evaluated by comparing the color developed in the magnetic system vs serum titration on classic ELISA. Negative samples developed no visual color in magnetic bead system (Figure 2A), whereas mild and severe COVID-19 serum samples were visually distinguished from negative samples (Figure 2A). The color intensity clearly correlated with the serum titer observed using classic ELISA (Figure 2A,B). 
The raw OD obtained using classic ELISA and our method (using a cohort of 165 samples, 46 PCR-confirmed COVID-19 and 119 pre-pandemic negative controls) were highly correlated, Pearson's $R=0.91, p<0.0001$ (Figure 2C). Raw OD values obtained using serum from COVID-19 positive patients were not significantly different between classic ELISA and magnetic bead ELISA, $p=0.16$. On the other hand, the raw $\mathrm{OD}$ values of control samples were different between the methods, $p<0.0001$ (Figure 2C). The magnetic bead ELISA produced lower raw ODs than classic ELISA. Receiver operating characteristic (ROC) of these 165 samples indicated that the magnetic bead ELISA outperformed classic ELISA with area under the ROC curve of 0.97 vs 0.96 , respectively. Using the magnetic system, sensitivity of $97 \%$ could be reached at $100 \%$ specificity. Classic ELISA performed at $90 \%$ sensitivity at a cost of $100 \%$ specificity. The magnetic bead ELISA showed exceptionally low cross-reaction background in the negative group (mean raw $\mathrm{OD}=0.08 \pm 0.001$ ), thereby outperforming classic ELISA in the ability to discriminate positive samples with low antibody titer (Figure 2C). Such low background allowed visual inspection of the results without significantly compromising accuracy (sensibility and specificity of $96 \%$ and $99 \%$ were obtained by visual inspection).

The magnetic-bead ELISA was further validated by analyzing 372 samples including 90 PCR-confirmed COVID19 and 282 negative controls. The method operated with an area under the ROC curve of 0.98 , 95\% sensitivity, and $99 \%$ specificity (further information on ROC interpretation is provided in the Supporting Information). Method cross validation was obtained by the team of operators in Germany (working independently from antigen preparation to testing). Using a different cohort of samples, which included 196 PCRconfirmed COVID-19 cases and 64 negative controls, the assay performed with area under the ROC curve of $0.93,83 \%$ sensitivity, and $98 \%$ specificity (Figure 2D). The differences in method performance between the two teams may be due to the fact that the cohort of positive samples analyzed in Germany consisted mostly of asymptomatic and nonhospitalized COVID-19 cases (97\%) whereas the cohort of positive cases used by the team in Brazil were mostly of hospitalized COVID-19 cases (58\%). Hospitalized cases are known to have increased IgG levels in comparison to mild COVID-19 cases. $^{12}$ Furthermore, about $10 \%$ of the same cohort of COVID-19 positive cases analyzed by the group in Germany did not have any detectable antibodies to multiple SARS-CoV-2 antigens as analyzed by the MagPlex system. ${ }^{11}$

Given that ROC analysis suggested similar raw OD cutoff from data sets obtained by the different laboratories, data sets were combined. The overall cohort consisted of 298 controls and 284 PCR-confirmed cases. The combined data set ROC had an area under curve of $0.98,87 \%$ sensitivity ( $81-90 \%$, $95 \% \mathrm{CI}$ ), and $99 \%$ specificity $(97-100 \%$, at $95 \% \mathrm{CI}$ ) (Figure S4). We believe that the combined data set represents seroconversion in the general population given that $81 \%$ of the COVID-19 cases are mild, and the combined data set had exactly $81 \%$ mild cases in the COVID-19 positive cohort. The overall method accuracy is in the same range as those obtained using Microplex to investigate IgG seroconversion for SARSCoV-2 using nucleocapsid protein as antigen. ${ }^{12}$ It worth mentioning that the signal of IgG reacting to SARS-CoV-2 N protein correlated well when data obtained using MagPlex and magnetic bead ELISA were compared (Pearson's $R=0.77, p<$ 0.0001 , Figure $2 \mathrm{E}$ ). Hence, the magnetic bead ELISA performs with accuracy equivalent to well established techniques at a fraction of the cost of consumables, instrumentation, time to deliver results, and volume of sample. The method can also be applied for IgM analysis (see Supporting Information).

We evaluated the ability of the method to discriminate COVID-19 convalescent cases at a point-of-care unit. For that, $5 \mu \mathrm{L}$ of capillary blood, collected using finger puncture, was directly diluted on the line B of the assay plates (see Figure 1). After the assay development, data was analyzed by visual inspection; cutoff was set applying a borderline high negative serum as control (Figure S5). By analyzing a cohort of 56 PCR confirmed COVID-19 cases (87\% nonhospitalized) and 47 negative controls (reporting no COVID-19 related symptoms since 2019) and area under the ROC curve of 0.96, 93\% sensitivity, and $100 \%$ specificity were obtained (Figure S5). Hence, the method is well suited for point-of-care analysis.

In this study, we used the format described in Figure $1 \mathrm{~A}$ with 12 samples being run simultaneously. However, the method produced equivalent data in multiple formats such as by running 48 samples simultaneously (Figure S6). The throughput of the process can be customized on demand, high throughput is simply achieved applying each step depicted in Figure 1 to occur on a full 96 well plate instead of a 12 well lane. Furthermore, the process can be automated using commercially available magnetic extractor devices.

\section{CONCLUSIONS}

In recent reviews and studies about immunological techniques for COVID-19 detection, including magnetic bead systems, all quantitative methods required more sample volume, time to deliver results, and/or instrumentation than the method we describe here. Furthermore, all quantitative methods require blood processing before the analysis. ${ }^{9,13,14}$ We believe that the simple, inexpensive, rapid, and quantitative method to detect SARS-CoV-2 seroconversion in humans described here may help to fast track COVID-19 cases especially in developing countries, where access to molecular testing is limited. The magnetic bead ELISA method described here is readily adapted to be used with any other $6 \times$ His tagged protein or peptide as antigen to track other diseases. We envisage that this method may substitute classic ELISA in near future.

\section{ASSOCIATED CONTENT}

\section{(5) Supporting Information}

The Supporting Information is available free of charge at https://pubs.acs.org/doi/10.1021/acssensors.0c02544.

Details of antigen purification, human samples, ELISA assays, method development and performance (PDF)

Video showing the magnetic bead capture and homogenization system (MP4)

\section{AUTHOR INFORMATION}

\section{Corresponding Author}

Luciano F. Huergo - Setor Litoral, Federal University of

Paraná (UFPR), Matinhos, PR 83260-000, Brazil;

(1) orcid.org/0000-0002-7587-9510; Phone: +55 41

996765856; Email: luciano.huergo@gmail.com

\section{Authors}

Khaled A. Selim - Interfaculty Institute for Microbiology and Infection Medicine, Eberhard Karls University of Tübingen, 72074 Tübingen, Germany 
Marcelo S. Conzentino - Setor Litoral, Federal University of Paraná (UFPR), Matinhos, PR 83260-000, Brazil

Edileusa C. M. Gerhardt - Biochemistry and Molecular Biology Department, Federal University of Paraná (UFPR), Curitiba, PR 80060-000, Brazil

Adrian R. S. Santos - Biochemistry and Molecular Biology Department, Federal University of Paraná (UFPR), Curitiba, PR 80060-000, Brazil

Berenike Wagner - Interfaculty Institute for Microbiology and Infection Medicine, Eberhard Karls University of Tübingen, 72074 Tübingen, Germany

Janette T. Alford - Interfaculty Institute for Microbiology and Infection Medicine, Eberhard Karls University of Tübingen, 72074 Tübingen, Germany

Nelli Deobald - Interfaculty Institute for Microbiology and Infection Medicine, Eberhard Karls University of Tübingen, 72074 Tübingen, Germany

Fabio O. Pedrosa - Biochemistry and Molecular Biology Department, Federal University of Paraná (UFPR), Curitiba, PR 80060-000, Brazil

Emanuel M. de Souza - Biochemistry and Molecular Biology Department, Federal University of Paraná (UFPR), Curitiba, PR 80060-000, Brazil

Meri B. Nogueira - Complexo Hospital das Clínicas, Federal University of Paraná (UFPR), Curitiba, PR 80060-000, Brazil

Sônia M. Raboni - Complexo Hospital das Clínicas, Federal University of Paraná (UFPR), Curitiba, PR 80060-000, Brazil

Dênio Souto - Chemistry Departament, Federal University of Paraná (UFPR), Curitiba, PR 80060-000, Brazil

Fabiane G. M. Rego - Post-Graduation Program in Pharmaceutical Sciences, Federal University of Paraná (UFPR), Curitiba, PR 80060-000, Brazil

Dalila L. Zanette - Instituto Carlos Chagas - FioCruz, Curitiba, PR 81310-020, Brazil

Mateus N. Aoki - Instituto Carlos Chagas - FioCruz, Curitiba, PR 81310-020, Brazil

Jeanine M. Nardin - Hospital Erasto Gaertner, Curitiba, PR 81520-060, Brazil

Bruna Fornazari - Hospital Erasto Gaertner, Curitiba, PR 81520-060, Brazil

Hugo M. P. Morales - Hospital Erasto Gaertner, Curitiba, PR 81520-060, Brazil

Vânia A. Borges - Secretaria Municipal de Saúde de Guaratuba, Guaratuba, PR 83280-000, Brazil

Annika Nelde - Clinical Collaboration Unit Translational Immunology, German Cancer Consortium (DKTK), Department of Internal Medicine, University Hospital Tübingen, 72076 Tübingen, Germany; Department of Immunology, Institute for Cell Biology and Cluster of Excellence iFIT (EXC2180) "Image-Guided and Functionally Instructed Tumor Therapies", Tübingen University, 72076 Tübingen, Germany

Juliane S. Walz - Clinical Collaboration Unit Translational Immunology, German Cancer Consortium (DKTK), Department of Internal Medicine and Department of Hematology, Oncology, Clinical Immunology and Rheumatology, University Hospital Tübingen, 72076 Tübingen, Germany; Department of Immunology, Institute for Cell Biology and Cluster of Excellence iFIT (EXC2180)

"Image-Guided and Functionally Instructed Tumor

Therapies", Tübingen University, 72076 Tübingen, Germany
Matthias Becker - NMI Natural and Medical Sciences Institute at the University of Tübingen, 72770 Tübingen, Germany

Nicole Schneiderhan-Marra - NMI Natural and Medical Sciences Institute at the University of Tübingen, 72770 Tübingen, Germany

Ulrich Rothbauer - NMI Natural and Medical Sciences Institute at the University of Tübingen, 72770 Tübingen, Germany; Pharmaceutical Biotechnology, Tübingen University, 72076 Tübingen, Germany

Rodrigo A. Reis - Setor Litoral, Federal University of Paraná (UFPR), Matinhos, PR 83260-000, Brazil

Karl Forchhammer - Interfaculty Institute for Microbiology and Infection Medicine, Eberhard Karls University of Tübingen, 72074 Tübingen, Germany

Complete contact information is available at:

https://pubs.acs.org/10.1021/acssensors.0c02544

\section{Author Contributions}

L.F.H conceived and designed the study. K.A.S leaded the German team with support from L.F.H and K.F. L.F.H, K.S.A, B.W, J.T.A, N.D, M.S.C, E.C.M.G, A.R.S.S, and D.E.P.S collected data. M.B.N, F.G.M.R, S.M.R, D.L.Z., M.N.A., J. M.N., B.F., A.N, J.S.W, M.B, N.S-M, U.R, V.A.B, L.F.H., and H.M.P.M. contributed samples. F.O.P, E.M.S, K.F, L.F.H, and R.A.R contributed reagents and lab infrastructure. L.F.H analyzed the data. K.A.S analyzed the data in Germany. L.F.H, R.A.R, and K.F project management. L.F.H wrote the paper with the help of K.F and K.A.S.

\section{Notes}

The authors declare the following competing financial interest(s): Federal University of Parana UFPR is in the process of licensing out the assays to commercial entities and has filed for patent protection of the magnetic immunoassay process and a magnetic COVID-19 immunological test product.

\section{ACKNOWLEDGMENTS}

This work was supported by the Alexander von Humboldt foundation, UFPR, CNPq and CAPES. We would like to thank Heinz Grenzendorf for technical assistance. We are grateful to Tatielle Pricila Cintra dos Santos (UFPR-litoral) for the design of the magnetic holder. We acknowledge the infraestructural support of Prefeitura Municipal de Guaratuba and the Cluster of Excellence 'Controlling Microbes to Fight Infections' (EXC 2124) of the German research foundation (DFG).

\section{REFERENCES}

(1) Petherick, A. Developing Antibody Tests for SARS-CoV-2. Lancet 2020, 395, 1101.

(2) Long, Q. X.; Tang, X. J.; Shi, Q. L.; Li, Q.; Deng, H. J.; Yuan, J.; Hu, J. L.; Xu, W.; Zhang, Y.; Lv.; et al. Clinical and Immunological Assessment of Asymptomatic SARS-CoV-2 Infections. Nat. Med. 2020, 26, 1200.

(3) Long, Q. X.; Liu, B. Z.; Deng, H. J.; Wu, G. C.; Deng, K.; Chen, Y. K.; Liao, P.; Qiu, J. F.; Lin, Y.; Cai, X. F.; et al. Antibody Responses to SARS-CoV-2 in Patients with COVID-19. Nat. Med. 2020, 26, 845.

(4) Huang, A. T.; Garcia-Carreras, B.; Hitchings, M. D. T.; Yang, B.; Katzelnick, L. C.; Rattigan, S. M.; Borgert, B. A.; Moreno, C. A.; Solomon, B. D.; Trimmer-Smith, L.; et al. A Systematic Review of Antibody Mediated Immunity to Coronaviruses: Kinetics, Correlates of Protection, and Association with Severity. Nat. Commun. 2020, DOI: $10.1038 / s 41467-020-18450-4$. 
(5) Engvall, E.; Perlmann, P. Enzyme-Linked Immunosorbent Assay, Elisa. 3. Quantitation of Specific Antibodies by Enzyme-Labeled AntiImmunoglobulin in Antigen-Coated Tubes. J. Immunol. 1972, 109, 129-135.

(6) Huergo, L. F.; Conzentino, M. S.; Gerhardt, E. C. M.; Santos, A. R. S.; Pedrosa, F.; de, O.; Souza, E. M.; Nogueira, M. B.; Forchhammer, K.; Rego, F. G. M.; Raboni, S. M.; Reis, R. A. Magnetic Bead-Based ELISA Allow Inexpensive, Rapid and Quantitative Detection of Human Antibodies against SARS-CoV-2. medRxiv 2020, 1 DOI: 10.1101/2020.07.26.20162255.

(7) Liu, W.; Liu, L.; Kou, G.; Zheng, Y.; Ding, Y.; Ni, W.; Wang, Q.; Tan, L.; Wu, W.; Tang, S.; Xiong, Z.; Zheng, S. Evaluation of Nucleocapsid and Spike Protein-Based Enzyme-Linked Immunosorbent Assays for Detecting Antibodies against SARS-CoV-2. J. Clin. Microbiol. 2020, DOI: 10.1128/JCM.00461-20.

(8) Amanat, F.; Stadlbauer, D.; Strohmeier, S.; Nguyen, T. H. O.; Chromikova, V.; McMahon, M.; Jiang, K.; Arunkumar, G. A.; Jurczyszak, D.; Polanco, J.; et al. A Serological Assay to Detect SARSCoV-2 Seroconversion in Humans. Nat. Med. 2020, 26, 1033.

(9) Carter, L. J.; Garner, L. V.; Smoot, J. W.; Li, Y.; Zhou, Q.; Saveson, C. J.; Sasso, J. M.; Gregg, A. C.; Soares, D. J.; Beskid, T. R.; Jervey, S. R.; Liu, C. Assay Techniques and Test Development for COVID-19 Diagnosis. ACS Cent. Sci. 2020, 6, 591.

(10) Adams, E. R.; Ainsworth, M.; Anand, R.; Andersson, M. I.; Auckland, K.; Baillie, J. K.; Barnes, E.; Beer, S.; Bell, J.; Berry, T.; et al. Antibody Testing for COVID-19: A Report from the National COVID Scientific Advisory Panel [Version 1; Peer Review: Awaiting Peer Review]. Wellcome Open Res. 2020, 5, 139.

(11) Becker, M.; Strengert, M.; Junker, D.; Kerrinnes, T.; Kaiser, P. D.; Traenkle, B.; Dinter, H.; Haering, J.; Zeck, A.; Weise, F. Going beyond Clinical Routine in SARS-CoV-2 Antibody Testing - A Multiplex Corona Virus Antibody Test for the Evaluation of CrossReactivity to Endemic Coronavirus Antigens. medRxiv 2020, 1 DOI: $10.1101 / 2020.07 .17 .20156000$.

(12) den Hartog, G.; Schepp, R. M.; Kuijer, M.; GeurtsvanKessel, C.; van Beek, J.; Rots, N.; Koopmans, M. P. G.; van der Klis, F. R. M.; van Binnendijk, R. S. SARS-CoV-2-Specific Antibody Detection for Seroepidemiology: A Multiplex Analysis Approach Accounting for Accurate Seroprevalence. J. Infect. Dis. 2020, 222, 1452.

(13) Pokhrel, P.; Hu, C.; Mao, H. Detecting the Coronavirus (CoVID-19). ACS Sensors. 2020, 5, 2283.

(14) Cai, X.; Chen, J.; li Hu, J.; Long, Q.; Deng, H.; Liu, P.; Fan, K.; Liao, P.; Liu, B.; Wu, G.; et al. A Peptide-Based Magnetic Chemiluminescence Enzyme Immunoassay for Serological Diagnosis of Coronavirus Disease 2019. J. Infect. Dis. 2020, 222, 189. 